A. Bayo-Salas, J. Beerten, E. Prieto-Araujo, O. Gomis-Bellmunt, and D. Van Hertem, "Mitigation of harmonic interactions by the VSC-HVDC converter in variable networks," Proc. IET International Conference on AC and DC Power Transmission ACDC 2017, 11th ed., Manchester, UK, Feb. 14-16, 2017, 8 pages.

Digital Object Identifier: 10.1049/cp.2017.0036

URL (IET Digital Library):

https://digital-library.theiet.org/content/conferences/10.1049/cp.2017.0036

URL (IEEE Xplore Digital Library):

https://ieeexplore.ieee.org/document/7934965

(C) 2017 IET. This paper is a postprint of a paper submitted to and accepted for publication in Proc. IET International Conference on AC and DC Power Transmission 2017 and is subject to Institution of Engineering and Technology Copyright. The copy of record is available at IET Digital Library. 


\title{
Mitigation of harmonic interactions by the VSC-HVDC converter in variable networks
}

\author{
A. Bayo-Salas ${ }^{* \dagger}$, J. Beerten ${ }^{\dagger}$, E. Prieto-Araujo ${ }^{\ddagger}$, O. Gomis-Bellmunt ${ }^{\sharp \sharp}$, D. Van Hertem ${ }^{\dagger}$ \\ *Elia System Operator, Belgium, alejandro.bayosalas@elia.be, \\ $\dagger$ ELECTA, University of Leuven (KU Leuven) - EnergyVille, Belgium, \\ $\ddagger$ CITCEA-UPC, Universitat Politècnica de Catalunya, Barcelona, Spain, \\ \# IREC, Catalonia Institute for Energy Research, Barcelona, Spain
}

Keywords: Stability study, control interactions, VSC HVDC, active damping

\begin{abstract}
System stability can be affected by the interaction between the converter control and the network resonances. Current installations of VSC-HVDC converters have experienced instability issues due to this interaction and have triggered questions in industry and research such as the suitable methods to evaluate the interferences, the necessary models in order to determine them and the techniques to mitigate the effects. This paper first explains how to study the phenomena and to determine the frequency range of interactions. Several active damping techniques to mitigate the arising oscillations in networks with multiple and variable resonances are proposed. The techniques are implemented by means of different control loops and making use of the different control variables from measurements. Results from time-domain simulations show the satisfactory behaviour of the proposed controls.
\end{abstract}

\section{Introduction}

Power-electronic devices such as Voltage-Source Converters (VSC) operate in a wide range of frequencies and interact with the electromagnetic modes of the power system. As a consequence, a new type of stability problem has emerged in the power system known as harmonic stability, which causes power quality and dynamic issues $[1,2]$.

Harmonic interactions in high-voltage (HV) networks are currently a subject of great interest. First worldwide connections of multi-modular converters (MMC) to the HV network have experienced instability issues due to the interaction between the converter and resonances from the system [3,4]. In the literature, harmonic instabilities due to the interaction of converters with the submarine collector cables have been detected and studied [5-9]. Also, interactions between two parallel converters may lead to harmonic instabilities [10-12].

Harmonic interactions have been also studied in traction networks [13] and in distribution systems with inverters includ- ing an $L C L$ output filter [14]. In these installations, the harmonic instability is the result of the interaction between the $L C L$ filter resonance within the control plant. Furthermore, resonances from the network are less of a concern in these installations due to inherent damping in grids with lower $X / R$ ratio.

In the field of power electronics, methods to damp this type of $L C L$ resonance have been particularly proposed and are still under research. These active damping (AD) methods are divided into two categories: signal filters and virtual impedances. In the first class, signal filters are added in order to shape the loop of the control response in such a way that the stability margin at the resonant point is increased. These filters have been used for the damping of the $L C L$-filter resonance, such as lead-lags [15] or notch filters [16]; as well as in offshore installations, with the addition of a notch filter before the current controller [6]. In general, these methods are suitable for the damping of a well-determined resonant peak.

In the second class, virtual impedances emulate the expected converter behaviour, assuming this impedance would be present in the $L C L$ filter [17]. This virtual impedance is emulated through the control by means of adding a loop from the grid current feedback [18], capacitor current feedback [19-21] or capacitor voltage feed-forward [22,23]. For offshore applications where the converter has no output $L C L$ filter, a virtual resistor has been proposed [7]. It is implemented with the feedforward voltage and before the current controller and, thus, it is limited in bandwidth.

From the literature, we encounter the following questions and needs. First, previous works have studied the interactions by using the conventional $\pi$-equivalent representation of transmission lines, which has two main limitations: it is limited in bandwidth and, besides, the behaviour is not always accurate in the bandwidth because of the poor representation in the frequency domain [24]. Therefore, the arising oscillations are only representative for the simplified model and, thus, limited to the frequency range of the model simplification. However, the range up to these control interactions might occur is unknown and recent examples in the industry suggest that highfrequency interactions cannot be excluded beforehand [4]. For 


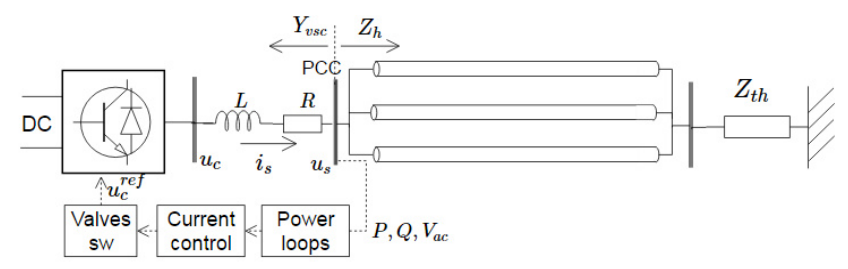

Fig. 1: Model of the AC system in study.

this reason, it is essential to determine the interactions using models accurately representing the frequency-dependent response and, based on the results, to implement the desired model simplifications for the analysis.

The second question relates to the resonance damping, previous works have been focused on the application for the output $L C L$ filter and offshore installations, where the resonance is determined by the VSC reactor and the equivalent capacitance and inductance of the cable. Therefore, the resonance is partially well characterized with a small uncertainty associated to network conditions. However, in a meshed grid scenario, resonances are more variable, uncertain and extended to a wider frequency range.

This paper deals with both previous questions. First, we explain how to conduct harmonic interaction studies and to determine the frequency range of interactions. Secondly, several active damping techniques are proposed and analysed with the objective of mitigating the oscillations in a network with multiple and variable resonances extended in a wider range.

\section{Study case}

A system composed of three identical transmission lines, a voltage-source converter and a Thévenin equivalent is presented in order to validate the effect of the significant changes in system topology on the appearance of resonances and the control robustness. This system is sketched in Fig. 1.

The transmission line is initially modelled with the universal line model [25], which is known to give the most accurate frequency-dependent representation of a line. In this paper, electromagnetic-transient simulations are performed in the software Matlab-SimPowerSystems (SPS). This software only has lumped-parameter line models available and, hence, does not accurately represent system dynamics in high frequencies. In order to overcome this issue, the line model is corrected by means of adding circuit components with the objective of fitting the frequency-dependant response of this model to the frequency-dependent response obtained with the universal line model [26, 27]. Fig. 2 shows the proximity of both frequency scans.

The Thévenin impedance is comprised between the values $Z_{t h, 1}=10 \angle 1.47^{\circ} \Omega$ and $Z_{t h, 2}=3 \angle 1.47^{\circ} \Omega$ with the objective of representing the uncertainty in the short-circuit impedance.

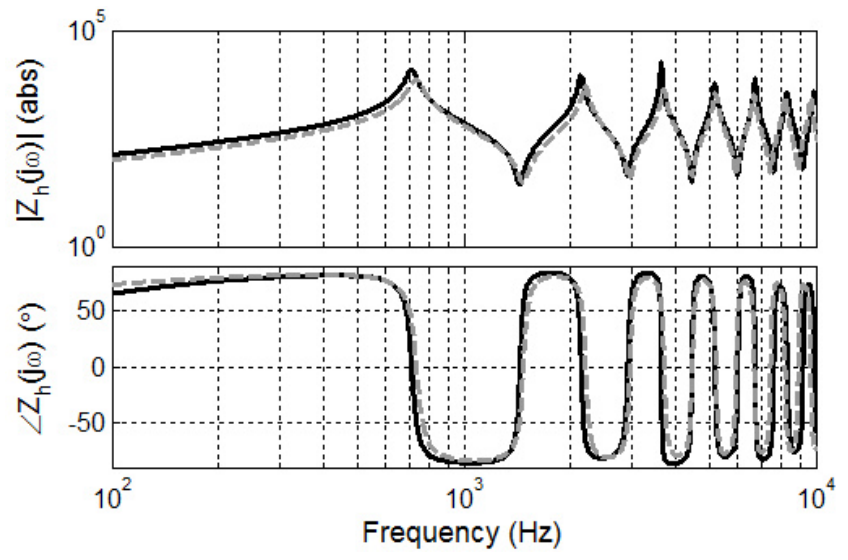

Fig. 2: Frequency sweep of the transmission line modelled with the universal line model (in grey dashed) and the implemented model in SPS (in solid) with an impedance termination.

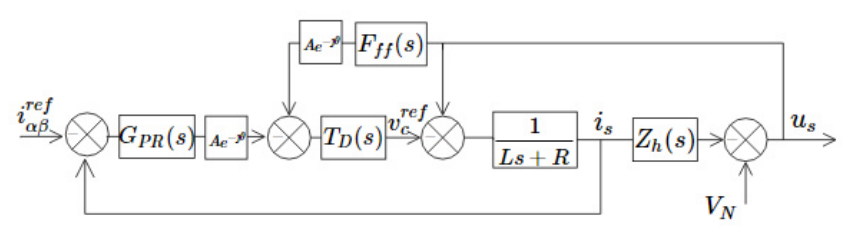

Fig. 3: VSC dynamic model and control.

This system is versatile in terms of bringing up different resonant points and, thus, validating the robustness of the proposed control to changes in the topology and resonances in a wider range. This way, the presented methodologies and techniques can also be extended to other networks.

The implemented VSC control and model is sketched in Fig. 3 . This VSC model contains the current controller compensator $G_{P R}$, the feed-forward filter $F_{f f}$, a time delay $T_{D}$, the reactor plant, and the Thévenin equivalent of the network $Z_{h}$. The different transfer functions and values are detailed in Appendix A. VSC dynamics are characterized by the frequencydependant admittance seen from the point of common coupling (PCC) $[28,29]$. In the specific case, the VSC admittance is calculated as,

$$
Y_{v s c}(s)=\frac{i_{s}}{u_{s}}=\frac{1-T_{D}(s) F_{f f}(s)}{L s+R+T_{D}(s) G_{P R}(s)}
$$

As the focus is on high-frequency dynamics, the operating point, which is related to power flow dynamics, does not have influence on the calculated $Y_{v s c}$ as it is also reflected in (1).

\section{Causes and frequency range of harmonic stability problems}

Harmonic instabilities arise from the interconnection of the converter to the network. From the control perspective, the 


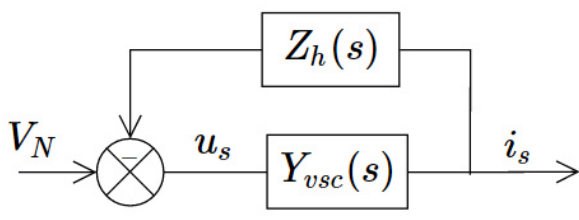

Fig. 4: Scheme of the dynamic interaction between the VSC and the network.

VSC interacts with the network through the input voltage disturbances and the output controlled current. This interference is dynamically sketched in the connection of two systems in Fig. 4 through the electrical variables. The oscillatory response is illustrated by the Bode diagram of the open loop transfer function between the VSC dynamics and the harmonic impedance of the network, i.e., $Y_{v s c} Z_{h}$ in Fig. 5. $Z_{h}$ is the frequency scan of a given network phase harmonic impedance. Dynamic interactions are determined by the small phase margin in the gain crossover frequencies of $Y_{v s c} Z_{h}$. More specifically, the small phase margin between $Y_{v s c}$ and the harmonic impedance of the network $Z_{h}$ leads to a sustained high frequency oscillation at $880 \mathrm{~Hz}$ seen in the time-domain response after a change in the network topology of an EMT simulation in Fig. 6.

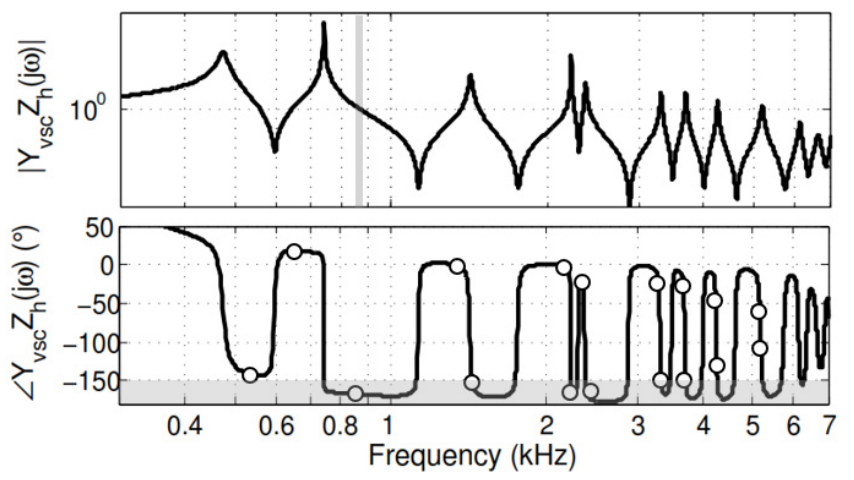

Fig. 5: Bode diagram of the open loop transfer function $Y_{v s c} Z_{h}$. Circles indicate the phase margin of the different gain crossovers which have an influence on the dynamic response. The dominant oscillatory behaviour is indicated by the line. The shaded area contains the different points which might lead to sustained oscillations.

The gain crossover leading to the sustained oscillation is dependent on the specific topology. After changes in the network, the resonant points vary and, hence, the arising harmonic interactions change as a consequence. For the study case in this paper, this specific gain crossover is situated in a range between 600 and $1000 \mathrm{~Hz}$, as shown in Fig. 7 and, therefore, the damping technique would need to be able to act and provide sufficient damping within this entire bandwidth. Because of this reason, strategies based on notch or lead-lag filters would not be able to provide it in such a wide bandwidth. Furthermore,

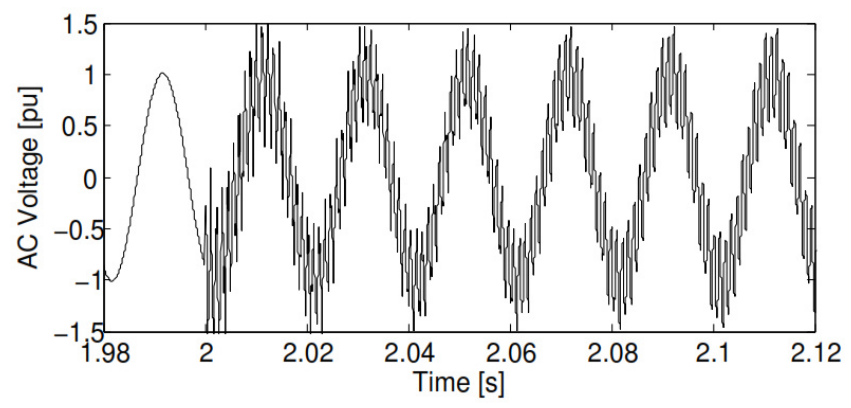

Fig. 6: EMT time-domain response after the connection of two of the transmission lines at $\mathrm{t}=2 \mathrm{~s}$.

the rest of gain crossover points shown in Fig. 5 consequently vary with the change in topology.

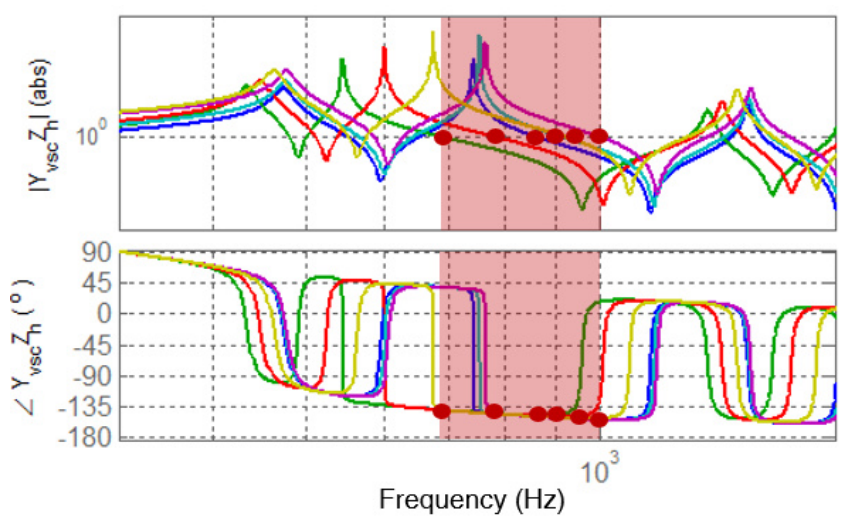

Fig. 7: Bode of the open loop transfer function $Y_{v s c} Z_{h}$ for all the network topologies in the defined system. The shaded area represents the frequency range of the gain crossover leading to the sustained oscillation for all the network topologies.

At this moment, there is no clear answer about the range of frequencies within which these interactions can typically occur and is still a subject to study. As a common approach, studies request data up to the $50^{\text {th }}$ harmonic as a practice transferred from LCC harmonic studies. This range allows certain confidence in the frequency-dependant modelling of the network as, e.g., parasitic effects of transformers start to appear after this range.

Similarly, the frequency range of interactions can be determined by the range where gain and phase crossovers in the open loop $Y_{v s c} Z_{h}$ present sufficient phase and gain margin respectively or, being more secure, they are non-existent ${ }^{1}$. As indicated in Fig. 5, gain crossovers disappear at $4 \mathrm{kHz}$ and show a sufficient phase margin after $2.5 \mathrm{kHz}$, implying that the network adds enough damping to oscillations beyond $2.5 \mathrm{kHz}$.

\footnotetext{
${ }^{1}$ Here it is important to estimate the inherent damping of the system in the high frequencies -skin effect, synchronous generators, etc-; otherwise the bandwidth of study would be unnecessarily increased and complicated
} 


\section{Active damping techniques}

The main objective of the implemented control is to increase the phase margin between the disturbance rejection response of the converter and the harmonic network impedance as explained in Section 3. In meshed AC networks, the active damping needs to be able to mitigate interactions with resonances in uncertain and variable conditions where low-damped electromagnetic modes can appear in a wider range.

The damping techniques are implemented by means of using the different available feedback and feed-forward variables associated to the interaction, i.e., the controller current and the PCC voltage. In the paper, different techniques based on the use of the different variables are proposed.

\subsection{High-pass filter}

A straightforward way to increase the phase margin is to add a derivative term in the voltage feed-forward. In order to not to affect the noise rejection, a $2^{\text {nd }}$-order high-pass filter $H_{h p f}$ with $\omega_{c}=2 \pi 600 \mathrm{rad} / \mathrm{s}$ is added after the feed-forward filter. $H_{h p f}$ is added to the output of the reference modulation voltage as shown in Figure 8. The filter could be substituted by a bandpass filter if, instead, it is desired to only add damping within a specific range of frequencies.

The equivalent admittance $Y_{v s c}$ including the addition of the high pass filter is calculated as,

$$
Y_{v s c}=\frac{i_{s}}{u_{s}}=\frac{1-T_{D}(s) F_{f f}(s)\left(H_{h p f}(s)+1\right)}{L s+R+T_{D}(s) G_{P R}(s)}
$$

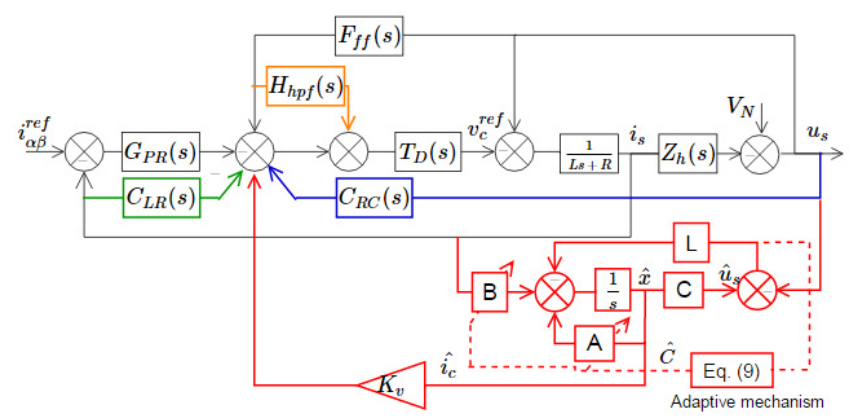

Fig. 8: Combined control diagram with the proposed active damping techniques: high-pass filter $H_{h p f}$ (in orange), virtual damping $C_{L R}$ from the converter current (in green), virtual damping $C_{R C}$ from the voltage (in blue) and Adaptive Kalman filter (in red).

\subsection{Virtual damping from the converter current feedback}

By multiplying the converter current feedback by $C_{L R}$ defined in eq. (3), the control emulates the addition of a $L R$ branch impedance $Z_{L R}=R_{v}+\jmath \omega L_{v}$ in parallel to the VSC series phase reactor. This virtual impedance has also been proposed to shape the $L C L$ plant in LV-inverter applications [18]. However, since the plant here is only the reactor and the resonance is caused by the interaction with the external network, the virtual impedance needs to be implemented in a different manner. The virtual impedance $Z_{L R}$ is sketched in Fig. 9 and the control diagram is shown in Fig. 8. The virtual impedance is designed with $L_{v}=R_{v}=1 \mathrm{pu}$. In order to implement $C_{L R}$ as a transfer function, the function in (3) is multiplied by a fast pole with $p=10^{-6}$.

$$
C_{L R}=-\frac{(L s+R)^{2}}{\left(L+L_{v}\right) s+\left(R+R_{v}\right)} \frac{1}{p s+1}
$$

The equivalent VSC admittance is calculated as,

$$
Y_{v s c}=\frac{i_{s}}{u_{s}}=\frac{1-T_{D}(s) F_{f f}(s)}{L s+R+T_{D}(s) G_{P R}(s)+T_{D}(s) C_{L R}(s)}
$$

\subsection{Virtual damping from the voltage feed-forward}

Alternatively, the voltage feed-forward can be used in order to add a virtual shunt $R C$ branch at the PCC, similar as the approach for the $L C L$-resonance damping presented in [21,23]. This virtual impedance needs to be implemented in a different manner because the plant is not an $L C L$ filter and the parameters defining the resonance are not available. Here, the feed-forward voltage is multiplied $C_{R C}$ which is implemented as (5). This loop emulates a shunt virtual impedance, $Z_{R C}=R_{v}+\frac{1}{\jmath \omega C_{v}}$ at the PCC as sketched in Fig. 9 and the control is implemented according to Fig. 8. The impedance is designed with $R_{v}=0.002 \mathrm{pu}$ and $C_{v}=0.38 \mathrm{pu}$. As in the previous case, the transfer function is implemented with a very fast pole.

$$
C_{R C}=\frac{R_{v} C_{v} s+1}{p s+1}
$$

The equivalent admittance $Y_{v s c}$ is calculated as,

$$
Y_{v s c}(s)=\frac{i_{s}}{u_{s}}=\frac{1-T_{D}(s)\left(F_{f f}(s)+Z_{R C}(s)\right)}{L s+R+T_{D}(s) G_{P R}(s)}
$$

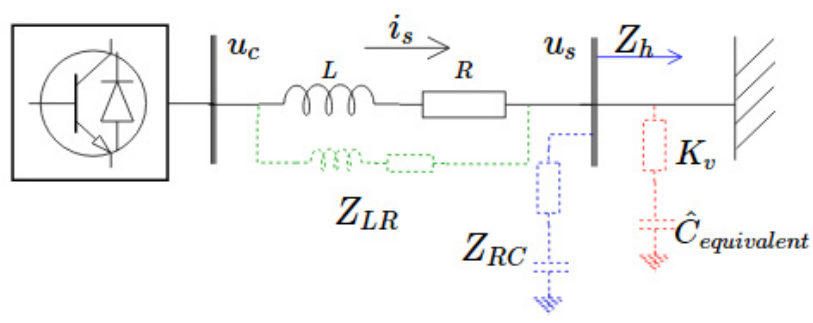

Fig. 9: Scheme of the emulated virtual impedances by means of control loops. 


\subsection{Virtual damping from the charging current: Adaptive Kalman Filter}

The damping of the $L C L$-filter resonance using the capacitive current feedback has been the most employed solution so far [19-21]. However, current sensors in the capacitor branch and the secondary inductive branch are required. In case of a real network, one cannot physically measure the charging current at the PCC and the current flowing at the network side. However, these could be estimated by means of a state observer using the existing measurements: the PCC voltage and the current through the phase reactor. To do this, one needs to estimate the parameters defining the state space representation of the system. In this case, the parameter is the equivalent capacitance characterizing the voltage dynamics. However, these parameters are dependent on the connected network and can thus not be defined in advance. Therefore, a control based on an adaptive Kalman filter which estimates the charging current and the equivalent capacitance at the node is proposed. The state estimation is used afterwards for the virtual damping. The loop emulates a resistor in series with the equivalent capacitance by multiplying the estimated current by a gain. The Kalman Filter is defined as follows,

$$
\begin{aligned}
s\left(\begin{array}{c}
\hat{u}_{s} \\
\hat{i}_{c}
\end{array}\right) & =\left(\begin{array}{cc}
0 & -\frac{1}{\hat{C}} \\
0 & 0
\end{array}\right)\left(\begin{array}{l}
\hat{u}_{s} \\
\hat{i}_{c}
\end{array}\right)+\left(\begin{array}{c}
\frac{1}{\hat{C}} \\
0
\end{array}\right) i_{s}-\mathbf{L}\left(\hat{u}_{s}-u_{s}\right) \\
\hat{u}_{s} & =\left(\begin{array}{ll}
1 & 0
\end{array}\right)\left(\begin{array}{c}
\hat{u}_{s} \\
\hat{i}_{c}
\end{array}\right)
\end{aligned}
$$

where $\hat{C}$ is the estimated capacitance from the adaptive mechanism defined below, $\mathbf{L}$ is the observer gain, and over-hat $\hat{x}$ denotes estimated variables and parameters. The adaptive mechanism is developed from the Lyapunov function candidate of the plant, defined as the energy stored $\frac{1}{2} C V^{2}$. The mechanism estimates $\hat{C}$ by a PI compensating the square error between the RMS estimated voltage and measured voltage as,

$$
\hat{C}=K_{P}\left(\left|\hat{U}_{s}\right|^{2}-\left.U_{s}\right|^{2}\right)+K_{I} \int_{0}^{t}\left(\left|\hat{U}_{s}\right|^{2}-\left.U_{s}\right|^{2}\right) d t
$$

Finally, the control scheme of the algorithm is presented in Fig. 8. The gain $K_{v}$ multiplying the estimated charging current $\hat{i}_{c}$ is equal to $0.5 \mathrm{pu}$.

The transfer functions are obtained from the state space model determined in (9), where $x=$ $\left[i_{\alpha} q_{1}^{\alpha} q_{2}^{\alpha} i_{\beta} q_{1}^{\beta} q_{2}^{\beta} u_{c \alpha} u_{c \beta} u_{s \alpha}^{f} u_{s \beta}^{f} \hat{u}_{s \alpha} \hat{u}_{s \beta} \hat{i}_{c \alpha} \hat{i}_{c \beta}\right]$, $u=\left[i_{\alpha}^{r e f} i_{\beta}^{r e f} u_{s \alpha} u_{s \beta}\right]$ and $y=\left[i_{\alpha} i_{\beta} \hat{i}_{c \alpha} \hat{i}_{c \beta}\right]$.

\section{Validation and Discussion}

The implementation of the different $\mathrm{AD}$ strategies causes a shape in the equivalent impedance from the converter. This loop shape leads to an increase of the phase margin in the crossover points of the open-loop $Y_{v s c} Z_{h}$. The open loop transfer function is calculated by multiplying the equivalent admittance, with the different damping techniques included, by the harmonic impedance of the network. The increase in phase margin is observed in Fig. 10. The different damping techniques are tested in an EMT time-domain simulation for all the different cases: for the three different topologies, for the two values of the Thévenin equivalent, $Z_{t h, 1}$ and $Z_{t h, 2}$, and for a VSC time delay $t_{d}$ equal to $1 \mu \mathrm{s}$ and $25 \mu \mathrm{s}$ in order to consider the associated uncertainty in VSC parameters. The cases more prone to high frequency oscillations happen when the time delay was higher and when the Thévenin impedance has the lowest value because resonances are less damped.

In the paper, only the time-domain response for the transient after the change in the topology from only one connected transmission line to the connection of three lines in parallel is presented here. Due to the instantaneous connection and energization of two transmission lines, this transient is considered the most extreme in the study case. In order to show the simulation for the most pessimistic case, the VSC time delay is equal to $25 \mu \mathrm{s}$ and the Thévenin impedance has the lowest value so that the resonances are less damped because there is a lower impedance for the termination. Time-domain responses of the phase voltage for the transient are shown in Fig. 11. In the figures, responses for each of the implemented controls is shown in comparison with the case without any damping control. All the responses show a satisfactory behaviour due to the successful damping of the sustained oscillation. However, for the high-pass filter control $(a)$ and the virtual damping from the converter current $(b)$, other oscillations appeared with a frequency around $2.2 \mathrm{kHz}$, also seen as a gain crossover in Fig. 5 . The oscillation disappears after $200 \mathrm{~ms}$ but demonstrates the limitation in bandwidth of the added damping. The observed dynamic confirms the importance to extend and determine the relevant frequency range of interaction.

Choosing the most suitable active damping technique will depend on a decision based on the control variable with which the active damping will be implemented. As an example, the use of the feed-forward voltage to implement the active damping might have an influence on the control response when there are large voltage deviations, suc as in weak grids. This influence is also dependent on other parameters of the overall control strategy. Therefore, the $\mathrm{AD}$ techniques influence on the overall response needs to be studied for the different VSC control strategies, for the different feed-forward characteristics, and for the different AC network characteristics.

\section{Conclusions}

Interactions between the voltage source converter and the network resonances cause stability issues. This paper focuses on the interactions leading from the connection of a converter to a variable network. Meshed HV networks give rise to several challenges for the mitigation of the stability problem such as the variation of the system resonances with the network topology and the low damping in high-voltage grids. Several control techniques with the objective of adding virtual damping are 


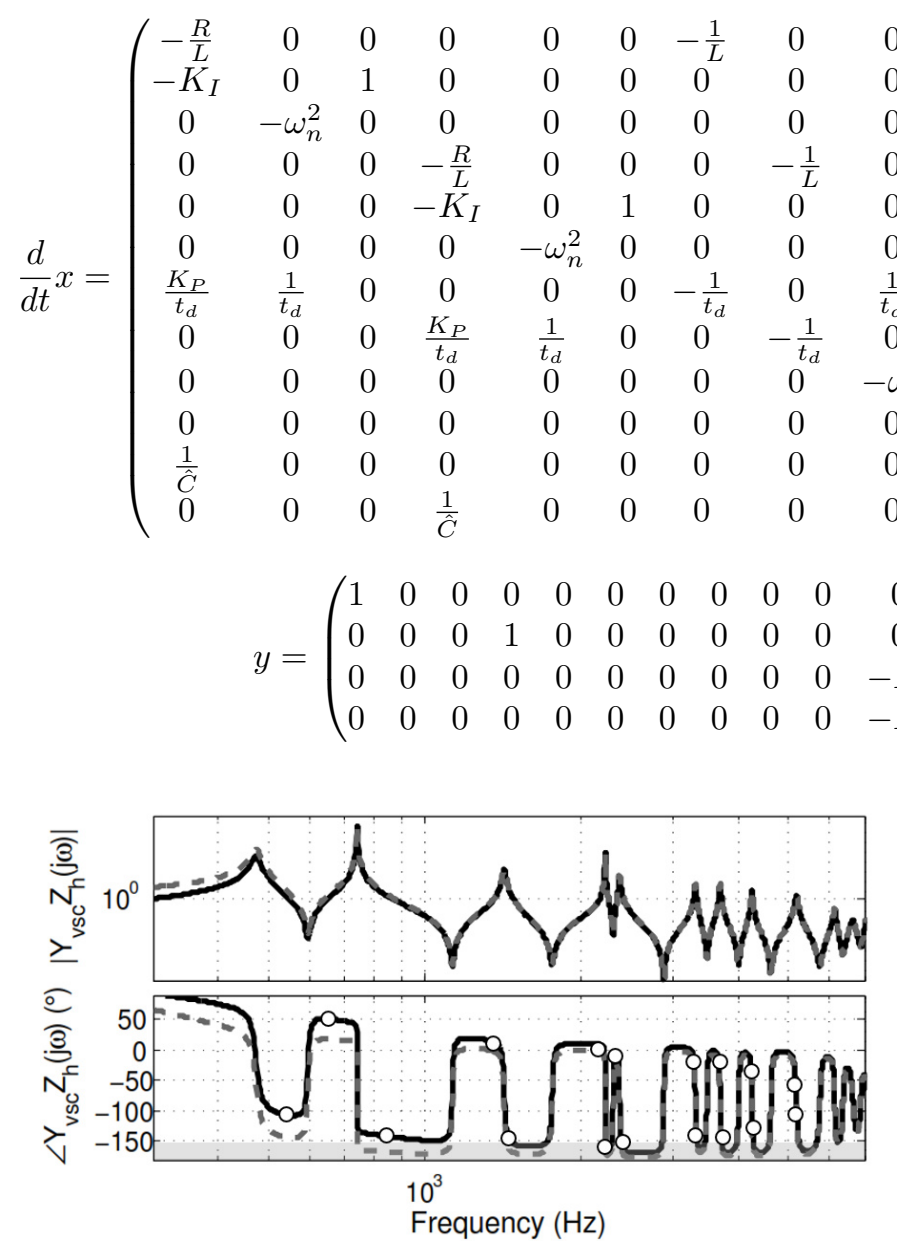

a) High-pass filter

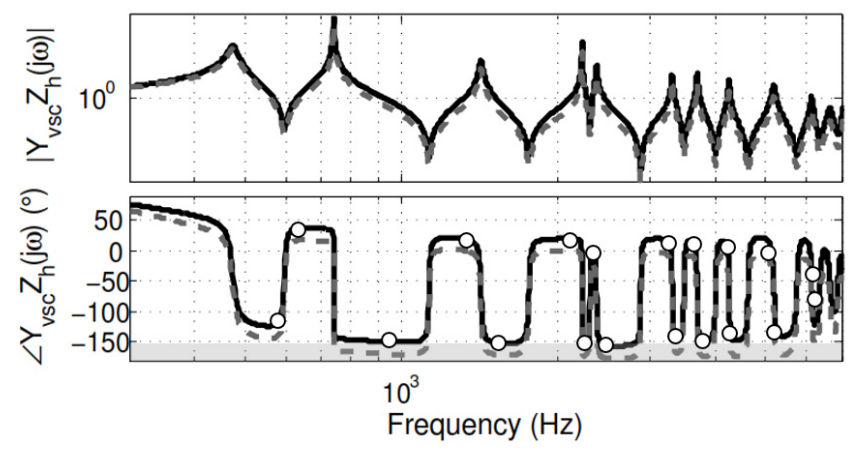

c) RC virtual damping

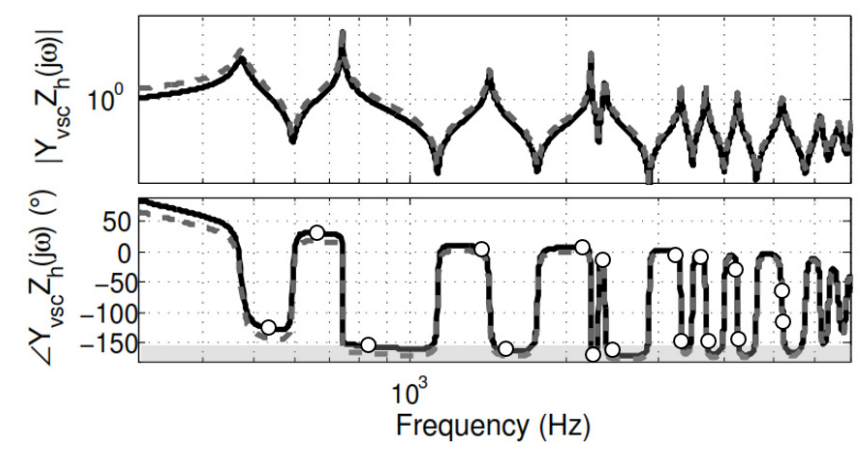

b) LR virtual damping

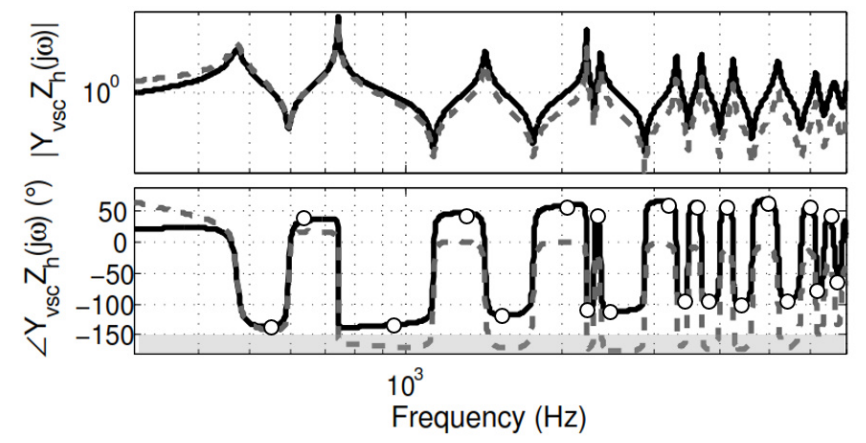

d) Adaptive Kalman filter

Fig. 10: Angle of the transfer function $Y_{v s c} Z_{h}$ for the different implemented strategies with active damping (in solid) and without $\mathrm{AD}$ (in dashed grey). $Z_{h}$ corresponds to the harmonic impedance for the given network after the studied transient (with the three transmission lines connected).

proposed. Results show the satisfactory response in mitigating the harmonic instability in the used variable network.

The paper demonstrates that these interactions can occur in a wide frequency range. For this reason, determining the range, as explained in the paper, needs to be conducted previous to the use of line model simplifications which might not give an accurate representation within the frequency range of interactions. The different strategies are based on the implementation of the active damping by using different control measurements depending on the convenience. The capability of adding damping over a wide frequency range is different between the tech- 

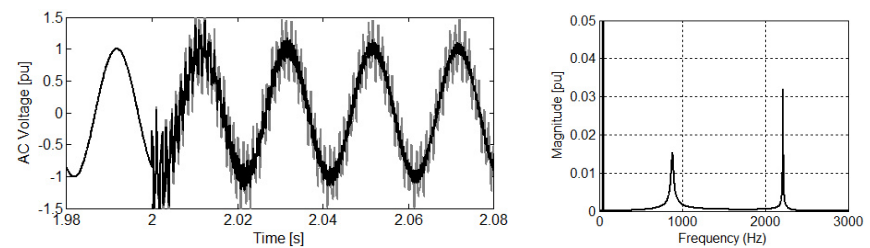

a) High pass filter
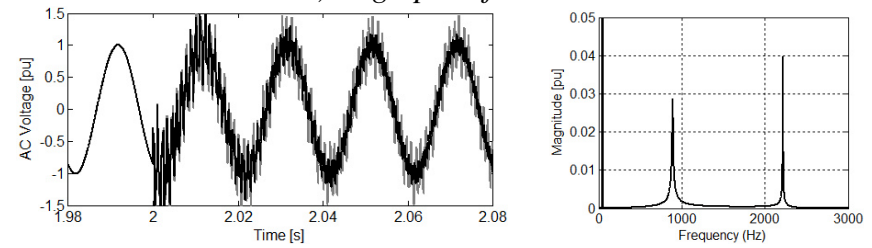

b) LR impedance
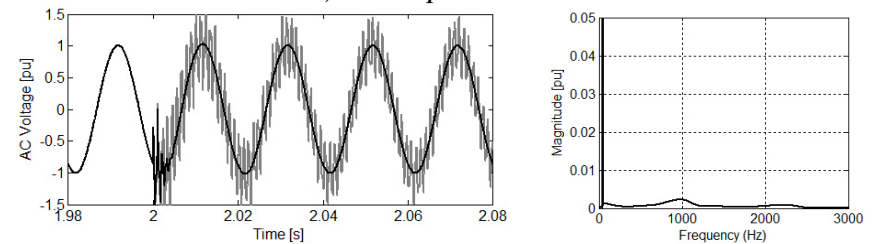

c) RC shunt impedance
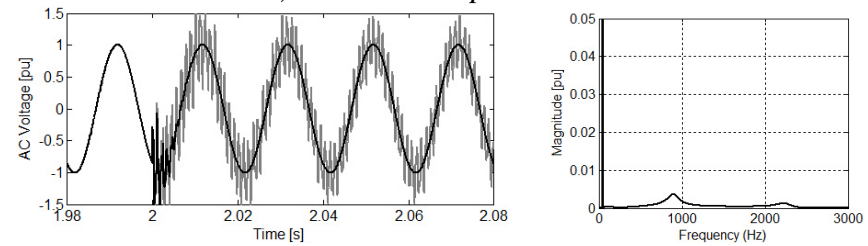

d) Adaptive Kalman filter

Fig. 11: Time-domain response of the phase voltage before (in grey) and after implementing the AD control (in black) and fft of the signal applied for the window between 2-2.3 s.

niques though all of them successfully mitigate the instability. In future work, the influence of the different techniques and their tuning in the overall dynamic behaviour will be studied.

\section{Acknowledgements}

The research leading to these results has received funding from the People Programme (Marie Curie Actions) of the European Union's Seventh Framework Programme FP7/2007-2013/ under REA grant agreement no. 317221, project title MEDOW. Jef Beerten is funded by a post-doctoral fellowship from the Research Foundation Flanders (FWO-Vlaanderen).

\section{References}

[1] P. Brogan, "The stability of multiple, high power, active front end voltage sourced converters when connected to wind farm collector system," in Proceedings in European Conference in Power Electronics and Applications, 2010, 6 pages.

[2] H. Enslin and P. J. Heskes, "Harmonic interaction between a large number of distributed power inverters and the distribution network," IEEE Transactions on Power Electronics, vol. 19, no. 6, pp. 1586-1593, November 2004.

[3] C. Buchhagen, C. Rauscher, A. Menze, and J. Jung, "BorWin1 - First Experiences with harmonics interactions in converter dominated grids," in International ETG Congress 2015 in Bonn., November 2015, 7 pages.

[4] H. Saad and (RTE). (2016, August) 'Performance Analysis of INELFE link with control replicas'. Workshop of 'Real-Time simulations to support installation and operation of HVDC/FACTS on transmission grids' durin Cigré Sessions 2016 in Paris. [Online]. Available: http://sites.google.com/site/hvdcreplicaworkshop/workshoppresentations [Accessed on 26th September 2016]

[5] L. H. Kocewiak, J. Hjerrild, and C. L. Bak, "Wind turbine converter control interaction with complex wind farm systems," IET Renewable Power Generation, vol. 7, no. 4, pp. 380-389, July 2013.

[6] J. Glasdam and L. H. Kocewiak, "Control System interaction in the VSC-HVDC Grid Connected Offshore Wind Power Plant," in Cigre B4 Simposium. Across borders - HVDC Systems and Market Integration in Lund., May 2015, 8 pages.

[7] S. Chaudhury, F. F. et R. Teodorescu, J. Guerrero, C. Bak, L. Kocewiak, and C. Jensen, "Harmonic Resonance in Wind Power Plants: Modeling, Analysis and Active Mitigation Methods," in Proceedings of the IEEE PowerTech Eindhoven 2015, 2015, 6 pages.

[8] M. Cheah-Mañé, J. Liang, N. Jenkins, and L. Sainz, "Electrical resonance instability study in HVDC-connected offshore wind power plants," in 2016 IEEE PES General Meeting, Boston, USA, 17-21st July 2016, 2016, pp. 1-7.

[9] L. Sainz, J. Mesas, L. Monjo, J. Pedra, and M. CheahMañé, "Electrical Resonance Instability Study in Wind Power Plants," in 10th Electric Power Quality and Supply Reliability Conference in Tallin (Estonia), 29-31st August 2016, p. 7 pages.

[10] A. Bayo-Salas, J. Beerten, J. Rimez, and D. Van Hertem, "Impedance-based stability assessment of parallel VSC HVDC grid connections," in Proc. in 11th International Conference on $A C$ and DC Power Transmission (ACDC 2015) Birmingham, February 2015, 9 pages.

[11] A. Bayo-Salas, J. Beerten, J. Rimez, and D. V. Hertem, "Analysis of control interactions in multi-infeed VSC HVDC connections," IET Generation, Transmission \& Distribution, vol. 10, no. 6, pp. 1336-1344, 2016.

[12] C.Wan, M.Huang, C. Tse, and X. Ruan, "Effects of Interaction of Power Converters Coupled via Power Grid: A DesignOriented Study," IEEE Transanctions on Power Electronics, vol. 30, no. 7, pp. 3589-3600, July 2015.

[13] E. Mollerstedt and B. Bernhardsson, "Out of control because of harmonics-an analysis of the harmonic response of an inverter locomotive," IEEE Control Systems, vol. 20, no. 4, pp. 70-81, August 2000.

[14] R. Teodorescu, M. Liserre, and P. Rodríguez, Grid Converters for Photovoltaic and Wind Power Systems, J. W. . Sons, Ed., 2011.

[15] J. Dannehl, M. Liserre, and F. Fuchs, "Filter-Based Active Damping of Voltage Source Converters With Filter," IEEE 
Transactions on Industrial Electronics, vol. 58, no. 8, pp. 3623-3633, August 2011.

[16] R. Peña-Alzola, M.Liserre, F. Blaabjerg, M. Ordonez, and T. Kerekes, "A self-commisioning Notch Filter for active damping in three-phase LCL-filter-based grid-tie converter," IEEE Transactions on Power Electronics, vol. 29, no. 12, pp. 6754-6761, December 2014.

[17] X. Wang, Y. W. Li, F. Blaabjerg, and P. C. Loh, "Virtualimpedance-based control for voltage-source and currentsource converters," IEEE Transactions on Power Electronics, vol. 30, no. 12, pp. 7019-7037, December 2015.

[18] X. Wang, F. Blaabjerg, and P. Loh, "Grid-Current-Feedback Active Damping of LCL Resonance in Grid-Connected Voltage-Source Converters," IEEE Transactions on Power Electronics, vol. 31, no. 1, pp. 213-223, January 2016.

[19] Y. Jia, J. Zhao, and X. Fu, "Converter Current Control of LCLfiltered grid-connected inverter mitigating grid voltage disturbance," IEEE Transactions on Power Electronics, vol. 29, no. 3, pp. 1532-1541, March 2014.

[20] M. Huang, X. Wang, P. Loh, and F. Blaabjerg, "Active Damping of LLCL-filter resonance based on LC-Trap Voltage or Current Feedback," IEEE Transactions on Power Electronics, vol. 31, no. 3, pp. 2337-2346, March 2016.

[21] X. Wang, F. Blaabjerg, and P. Loh, "Virtual RC Damping of LCL-Filtered Voltage Source Converters with extended selective harmonic compensation," IEEE Transactions on Power Electronics, vol. 30, no. 9, pp. 4726-4737, September 2015.

[22] V. Blasko and V. Kaura, "A novel control to acactive damp resonance in input LC filter of a three-phase voltage source converter," IEEE Transactions on Industrial Applications, vol. 33, no. 2, pp. 542-550, March 1997.

[23] Y. Yong, F. Gang, L. Jinjiun, Jiangfeng, and L. Tao, "A virtual RC active damping method in weak grid for three-level three-phase grid-connected inverters," in 2016 IEEE 8th International Power Electronics and Motion Control Conference (IPEMC-ECCE Asia, May 2016, pp. 1-7.

[24] J. Beerten, S. D'Arco, and J. Suul, "Frequency-Dependent Cable Modelling for Small-Signal Stability Analysis of VSCHVDC Systems," IET Generation Transmission \& Distribution, vol. 10, no. 6, pp. 1370-1381, 2016.

[25] A. Morched, B. Gustavsen, and M. Tartibi, "A universal line model for accurate calculation of electromagnetic transients on overhead lines and underground cables," IEEE Transactions on Power Delivery, vol. 14, no. 3, pp. 1032-1038, 1999.

[26] S. Kurokawa, F. Yamanaka, A. Prado, and J. Pissolato, "Inclusion of the frequency effect in the lumped parameters transmission line model: State space formulation," Electric Power Systems Research, vol. 79, pp. 1155-1163, 2009.

[27] A. R. J. de Araujo and S. Kurokawa, "Improving the Performance of the Lumped Parameters transmission Line Model by Using Analog Low-Pass Filters," in Proceedings in Internation Conference on Power Systems Transients (IPST 2015), Cavtat (Croatia) June 15-18, 2015, p. 8 pages.

[28] L. Harnefors, M. Bongiorno, and S. Lundberg, "Inputadmittance calculation and shaping for controlled voltagesource converters," IEEE Transactions on Industrial Electronics, vol. 54, no. 6, pp. 3323-3334, December 2007.
[29] J. Sun, "Impedance-based stability criterion for grid-connected inverters," IEEE Transactions on Power Electronics, vol. 26, no. 11, pp. 3075-3078, November 2011.

[30] R. Bojoi, G. Griva, V. Bostan, M. Guerriero, F. Farina, and F. Profumo, "Current control strategy for power conditioners using sinusoidal signal integrators in synchronous reference frame," IEEE Transactions on Power Electronics, vol. 20, no. 6, pp. 1402-1412, November 2005.

\section{A VSC control and model}

The phase reactor is modelled by the equivalent phase impedance including the arm and leakage inductance as,

$$
X=\frac{X_{a r m}}{2}+X_{t r}
$$

Total values are $X=0.14 \mathrm{pu}$ and $R=0.004 \mathrm{pu}$. The transfer functions in Fig. 3 are defined below,

- Proportional-resonant controller. The controller is tuned with a time constant of $1 \mathrm{~ms}$

$$
G_{P R}(s)=K_{P}+\frac{K_{I} s}{s^{2}+\omega_{N}^{2}}
$$

- Time Delay with $t_{d}$ between $1 \div 25 \mu \mathrm{s}$.

$$
T_{D}(s)=\frac{1}{t_{d} s+1}
$$

- Feed-forward filter. The cut-off frequency $\omega_{c}$ is $2 \pi 350 \mathrm{rad} / \mathrm{s}$.

$$
F_{f f}(s)=\frac{\omega_{c}}{s+\omega_{c}}
$$

One of the control complexities of controlling in $\alpha \beta$ is the imperative need of compensating all the changes in magnitude and phase in the signal caused by delays or filters. These deviations need to be corrected in order to align all signals in phase and magnitude; otherwise, the deviation will have a negative impact on the reference tracking.

$A e^{\jmath \theta}$ represents the change in amplitude and angle on the signal. Concretely, the correction in angle after the compensator is done for the delay and it has been implemented as in [30]; after the feed-forward filter, the correction is compensating the change in magnitude and angle due to the feed-forward filter. 